\title{
Scattering forces on magneto-dielectric particles and the electromagnetic momentum density
}

\author{
Manuel I. Marqués ${ }^{1,2 *}$, and Juan Jose Sáenz ${ }^{2,3}$ \\ ${ }^{1}$ Departamento de Física de Materiales, Universidad Autónoma de Madrid, 28049, Madrid, Spain \\ ${ }^{2}$ Instituto de Ciencia de Materiales Nicolás Cabrera and Instituto de Física de la Materia Condensada (IFIMAC), \\ 28049, Madrid, Spain \\ ${ }^{3}$ Departamento de Fśica de la Materia Condensada, Universidad Autónoma de Madrid, 28049, Madrid, Spain \\ *corresponding author, E-mail: manuel . marques auam. es
}

\begin{abstract}
In this paper we analyse the non-conservative forces on magneto-dielectric particles in special configurations where the scattering force is not proportional to the average value of the Poynting vector. Based on these results we revisit the concept of the radiation pressure exerted by the electromagnetic radiation on a small particle.
\end{abstract}

\section{Introduction}

After the pioneering experimental work by Ashkin and coworkers [1], the mechanical action of light on small particles has been extensively used to trap and manipulate small particles $[2,3,4]$ or to induce significant optical forces between particles $[5,6,7]$. Some recent proposals like the optical tractor beams $[8,9,10,11,12,13,14]$, have renewed the interest in this field.

Small dielectric particles compared with the radiation wavelength develop an electric dipole moment that is drawn by intensity gradients and that competes with the radiation pressure due to the momentum transferred from the photons to the particle. Actually, light forces on small dielectric particles are described as the sum of the dipole or gradient force and the radiation pressure or scattering force $[15,16]$.

Radiation pressure is usually considered proportional to the Poynting vector. However this is not the case for inhomogeneous waves. For non-homogeneous waves there is also an additional contribution to the scattering force [17], which has been shown to be a non-conservative force proportional to the curl of the spin angular momentum of the light field [18]. Actually, this curl term maybe interpreted not like an extra force, but like the absence of the spin component in the momentum transferred, which is then simply given by the orbital component of the Poynting vector [19, 20, 21].

This second contribution to the scattering force may be essential to understand the behaviour of nano particles under non-homogeneous waves. For example both, forces coming from the Poynting vector and spin forces, must be considered in order to understand the scattering forces in the focal volume of microscope objectives [22, 23, 24, 25].
These non-homogeneous light fields may be also produced by an appropriate manipulation of interfering laser beams. For example, it is possible to generate local vortices and complex Poynting vector patterns [26] leading to complex dynamics of the nanoparticles [27, 28, 29] and tractor properties [14]. These interesting field configurations are usually based on the interference between linearly polarized fields. Recently, the forces on a small dipolar particle and the electromagnetic momentum density in a configuration consisting in two perpendicular circularly polarized stationary waves have been analysed [30]. The field distribution for this particular configuration shows regions in which electric and magnetic fields are parallel, corresponding to a null Poynting vector. Although the average value of the momentum density, proportional to the Poynting vector, is zero in these regions, a non-negligible scattering force, due to light's spin force, was found.

In this paper we will review this result and we will extend it to the case of magneto dielectric particles. We will also discuss about the value of the linear momentum transferred to the particle, based on the total scattering forces.

\section{Scattering forces with null Poynting vector in dielectric nanoparticles}

Let us consider a small dielectric particle and let us treat it as a point dipole proportional to the external field, $\mathbf{p}=$ $\epsilon_{0} \alpha \mathbf{E}$ where $\alpha(\omega)=\alpha^{\prime}(\omega)+i \alpha^{\prime \prime}(\omega)$ is the complex polarizability and $\omega$ the frequency of the electromagnetic field. The total time averaged scattering force may be written as the sum of two terms; a scattering force proportional to the Poynting vector and a scattering force proportional to the curl of the spin angular momentum of light [18]:

$$
\langle\boldsymbol{F}\rangle=\sigma\left\{\frac{1}{c}\langle\boldsymbol{S}\rangle\right\}+\sigma\left\{c \boldsymbol{\nabla} \times\left\langle\boldsymbol{L}_{S}\right\rangle\right\}
$$

where $\sigma=k \alpha^{\prime \prime}$ is the particle's extinction cross section. The time averaged Poynting vector is,

$$
\langle\boldsymbol{S}\rangle=\operatorname{Re}\left\{\mathbf{E} \times \mathbf{H}^{*}\right\} / 2
$$


and the last term on the scattering force, related to the spin density of the light field, is given by,

$$
\left\langle\boldsymbol{L}_{S}\right\rangle=\epsilon_{0}\left\{\mathbf{E} \times \mathbf{E}^{*}\right\} /(4 \omega i)
$$

It is worth to mention that the scattering force given by Eq. 1 applies not only to "point particles" but also to finite size particles whose size is much smaller than the wavelength. As shown in Ref. [31], Eq. 1 can be directly derived from the Maxwell stress tensor assuming that the external exciting fields $\mathbf{E}$ and $\mathbf{H}$ are slowly varying in a region of the order of the particle size and that the scattered field "outside" the particle can be approximated as the field emitted from a dipole.

Let us analyse the average value of the scattering force when the small dielectric particle is placed in a electromagnetic field consisting in two perpendicular circularly polarized stationary waves with wavelength $\lambda$ and wave vector of modulus $k=2 \pi / \lambda$, propagating in the $\mathrm{X}$-Y plane and with a different of phase of $\pi / 2$.

$$
\begin{aligned}
& \boldsymbol{E}_{\mathbf{1}}=\left(e^{i k x}-e^{-i k x}\right) \frac{1}{\sqrt{2}}(0,1, i) \\
& \boldsymbol{E}_{\mathbf{2}}=\left(e^{i k y}-e^{-i k y}\right) \frac{i}{\sqrt{2}}(1,0, i)
\end{aligned}
$$

The value of the total scattering force is given by [30]

$$
\begin{aligned}
& \left\langle F_{x}\right\rangle=-\alpha^{\prime \prime} k \epsilon_{0}[\cos (k x) \sin (k y)] \\
& \left\langle F_{y}\right\rangle=\alpha^{\prime \prime} k \epsilon_{0}[\cos (k y) \sin (k x)] \\
& \left\langle F_{z}\right\rangle=0
\end{aligned}
$$

while the Poynting vector is given by [30]

$$
\begin{gathered}
\left\langle S_{x}\right\rangle=-\epsilon_{0} c[\cos (k x) \sin (k y)+\sin (k x) \cos (k y)] \\
\left\langle S_{y}\right\rangle=-\left\langle S_{x}\right\rangle \\
\left\langle S_{z}\right\rangle=0
\end{gathered}
$$

In points given by

$$
y=-x \pm \frac{m \lambda}{2}
$$

being $m$ an integer number, the electric and magnetic fields are parallel, implying an average value of the Poynting vector equal lo zero. However, the total scattering force on these points is non zero.

If the scattering force is considered to be a momentum exchange with the light field then, in this points, we have an anomalous situation where the scattering force is non zero but the electromagnetic linear momentum density, proportional to Ponting vector, is zero. Let us analyse if this anomalous situation also holds in the case of small magnetic particles.

\section{Scattering forces with null Poynting vector in magneto-dielectric nanoparticles}

The optical scattering force on magnetic particles has been analysed in Ref. [31, 32]. If the magnetic moment induced by the electromagnetic field is given by $\mathbf{m}=\left(\alpha_{m} / \mu_{0}\right) \mathbf{B}$ then the total scattering optical force is

$$
\langle\boldsymbol{F}\rangle=\sigma_{m}\left\{\frac{1}{c}\langle\boldsymbol{S}\rangle\right\}+\sigma_{m}\left\{c \boldsymbol{\nabla} \times\left\langle\boldsymbol{L}_{S M}\right\rangle\right\}
$$

where $\sigma_{m}=k \alpha_{m}^{\prime \prime}$ is the particle's magnetic extinction cross section and the magnetic spin density of the light field is given by,

$$
\left\langle\boldsymbol{L}_{S M}\right\rangle=\left(1 / \mu_{0}\right)\left\{\mathbf{B} \times \mathbf{B}^{*}\right\} /(4 \omega i)
$$

The Poynting vector has not changed with respect to the previous case but, in order to calculate the total scattering force on the magnetic particle, we must calculate the new force due to the curl of the magnetic spin density of the light field. This curl of the spin density is given by

$$
\begin{aligned}
\nabla \times\left.\left\langle\boldsymbol{L}_{S M}\right\rangle\right|_{x} & =\left(\epsilon_{0} / c\right)[\cos (k x) \sin (k y)] \\
\nabla \times\left.\left\langle\boldsymbol{L}_{S M}\right\rangle\right|_{y} & =-\left(\epsilon_{0} / c\right)[\cos (k y) \sin (k x)] \\
\nabla \times\left.\left\langle\boldsymbol{L}_{S M}\right\rangle\right|_{z} & =0
\end{aligned}
$$

Once we know the spin density we may calculate the total scattering force:

$$
\begin{aligned}
\left\langle F_{x}\right\rangle & =-\alpha_{m}^{\prime \prime} k \epsilon_{0}[\cos (k y) \sin (k x)] \\
\left\langle F_{y}\right\rangle & =\alpha_{m}^{\prime \prime} k \epsilon_{0}[\cos (k x) \sin (k y)] \\
\left\langle F_{z}\right\rangle & =0
\end{aligned}
$$

Again it is possible to find regions where the Poynting vector is zero but the average value of the scattering forces, due to the existence of a magnetic spin density with a value of the curl different from zero, is not null (see Figure 1).

The scattering forces for magnetic and dielectric particles are different but, in this particular case, the dynamical trend of both kind of particles, dielectric and magnetic, is going to be the same. We should say that this is not always the case. For example, in the case of linearly polarized beams, a s-polarization implies a null value of the spin force for the dielectric particle and a value different from zero for the magnetic particle. And, for p-polarization, the situation is just the opposite [18].

For small particles with a non- negligible electric and magnetic induced dipole there is also an extra contribution to the force given by the interaction between both dipoles, magnetic and electric [31, 32]. But, for pure scattering forces (coming solely from the imaginary parts of the polarizability, $\alpha^{\prime}=\alpha_{m}^{\prime}=0$ ) the contribution from this mixing term is simply proportional to the average value of the Poynting vector [33]. 


\section{Revisiting the electromagnetic linear momentum transferred to a small particle}

By summarizing the results presented in this paper, we may say that, even with a null average value of the Poynting vector, a dielectric particle experiences a pure scattering force given by

$$
\langle\boldsymbol{F}\rangle=\sigma\left\{c \boldsymbol{\nabla} \times\left\langle\boldsymbol{L}_{S}\right\rangle\right\}
$$

while a magnetic particle, with zero Poynting vector, experiences a scattering force given by

$$
\langle\boldsymbol{F}\rangle=\sigma_{m}\left\{c \boldsymbol{\nabla} \times\left\langle\boldsymbol{L}_{S M}\right\rangle\right\}
$$

Then, it seems natural to redefine the radiation pressure exerted over a small particle as the sum of the forming contribution coming from the Poynting vector $\left(\boldsymbol{S} / c^{2}\right)$ and the curls of the electric and magnetic spin densities [30], i.e. for the dielectric particle,

$$
\left\langle\boldsymbol{p}_{\boldsymbol{e}}\right\rangle=\frac{\langle\boldsymbol{S}\rangle}{c^{2}}+\left(\boldsymbol{\nabla} \times\left\langle\boldsymbol{L}_{S}\right\rangle\right)
$$

and for the magnetic particle,

$$
\left\langle\boldsymbol{p}_{\boldsymbol{m}}\right\rangle=\frac{\langle\boldsymbol{S}\rangle}{c^{2}}+\left(\boldsymbol{\nabla} \times\left\langle\boldsymbol{L}_{S M}\right\rangle\right)
$$

The terms added are solenoidal so they have no influence on the conservation laws obtained from Maxwell equations.

The average value of the electromagnetic momentum transferred to the dielectric particle may also be written as

$$
\left\langle\boldsymbol{p}_{\boldsymbol{e}}\right\rangle=\frac{i \epsilon_{0}}{4 \omega} \sum_{i=x, y, z}\left(E_{i} \nabla E_{i}^{*}-E_{i}^{*} \nabla E_{i}\right)
$$

while for the magnetic particle it may be written as

$$
\left\langle\boldsymbol{p}_{\boldsymbol{m}}\right\rangle=\frac{i}{4 \omega \mu_{0}} \sum_{i=x, y, z}\left(B_{i} \boldsymbol{\nabla} B_{i}^{*}-B_{i}^{*} \nabla B_{i}\right)
$$

Note how both expressions are the natural vectorial extension of the density of linear momentum for a scalar field $\Psi$ in quantum mechanics [30],

$$
\frac{i \hbar}{2}\left(\Psi \nabla \Psi^{*}-\Psi^{*} \nabla \Psi\right)
$$

A possible value for a instantaneous electromagnetic linear momentum density, with average value like the one obtained in 26 , is given by

$$
\boldsymbol{p}_{\boldsymbol{e}}=\epsilon_{0} \sum_{i=x, y, z} E_{i} \nabla A_{i}
$$

being $\boldsymbol{A}$ the gauge invariant transverse vector potential [34], while and average value like 27 is obtained with

$$
\boldsymbol{p}_{\boldsymbol{m}}=-\frac{1}{\mu_{0} c} \sum_{i=x, y, z} B_{i} \nabla C_{i}
$$

being $C$ the dual transformation of the vector potential $\boldsymbol{A}[35,36]$. These terms are usually named as the orbital part of the electromagnetic linear momentum density.

Interestingly, by considering the transverse gauge [34] for the free electromagnetic fields, the following linear momentum density $\hat{\boldsymbol{p}}$ is obtained directly from the canonical stress energy tensor [37]

$$
\hat{p}_{i}=\frac{S_{i}}{c^{2}}+\epsilon_{0} \nabla\left(\boldsymbol{E} A_{i}\right)
$$

and it is easy to show that in vacuum

$$
\hat{\boldsymbol{p}}=\boldsymbol{p}_{\boldsymbol{e}}
$$

Then, the momentum transferred to the dielectric particle, because of the interaction with radiation, is equal to the linear momentum density derived for the electromagnetic fields in vacuum, when the canonical stress energy tensor is considered. The linear momentum transferred to the magnetic particles $\boldsymbol{p}_{\boldsymbol{m}}$ is also obtained from the canonical stress energy tensor, but in this case, the dual electromagnetic fields must be considered $[35,36]$.

So we could be tempted to consider the linear momentum density, obtained from the canonical stress energy tensor, as the linear momentum density carried by the electromagnetic radiation in free space. However, we must be very careful because the canonical stress energy tensor has a major drawback, it is not symmetric. This asymmetry has two main consequences: First, the instantaneous time variation of the electromagnetic energy density $(\nabla \boldsymbol{S})$ is not longer proportional to the instantaneous divergence of linear momentum density $\left(c^{2} \nabla \hat{\boldsymbol{p}}\right)$. Second, for a non symmetric stress energy tensor the electromagnetic angular momentum is, in general, not conserved [37]. Although we should point out that the total angular momentum derived from the calculation of $\boldsymbol{r} \times \hat{\boldsymbol{p}}$, usually known as the orbital angular momentum of light, is conserved [38].

\section{Conclusions}

We have analysed the scattering force for crossed circularly polarized standing waves where the field distribution shows regions with parallel electric and magnetic fields, corresponding to a null Poynting vector. We have shown that, although the momentum density proportional to the Poynting vector is zero in these regions, there are scattering forces acting on small dielectric particles due to light's spin force. We have extended these results to small particles presenting both, electric and magnetic responses. We have discussed the intriguing interplay between the momentum and spin 


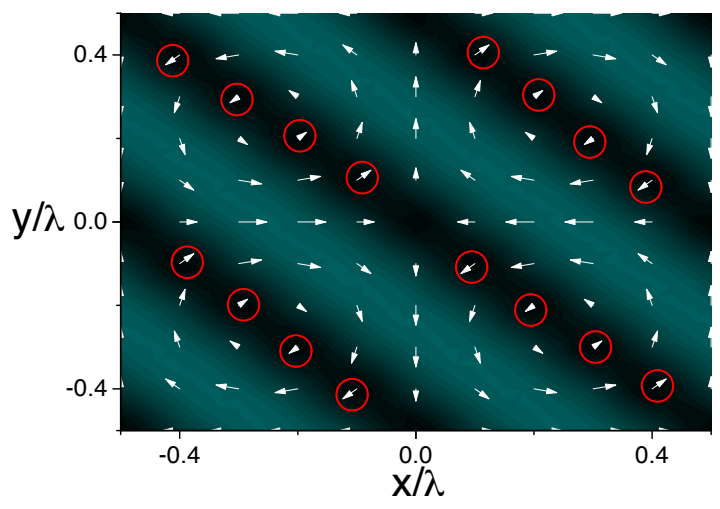

Figure 1: Scattering force on a magnetic particle for the configuration consisting in two perpendicular, circularly polarized, stationary waves with wavelength $\lambda$ propagating in the $\mathrm{X}-\mathrm{Y}$ plane and with a difference on the phase $\phi=\pi / 2$. The colour map represents the magnitude of the Poynting vector (darker for lower values). Red circles highlight the scattering forces which are different from zero in a region with null value of the Poynting vector.

densities and the actual force on magneto-dielectric particles. The optical force suggests a new definition of the radiation pressure exerted by light on a small particle, proportional to the full scattering forces.

Finally we would like to comment about the nonlocality of the phenomena reported in this work. As we already mention, the calculated scattering force applies also to finite size particles and, even though the Poynting vector vanishes at particular points in space, it does not vanish everywhere over the finite volume of the physical object. It would be interesting to seek for particular configurations where the Poynting vector is null everywhere over the volume of the object, while the scattering force is different from zero.

We hope this discussion will open intriguing basic new possibilities about the concept of the linear momentum transferred by the electromagnetic radiation to small magneto-dielectric particles.

\section{Acknowledgement}

This work was supported by the Spanish MEC through grants No. FIS2009-13430, FIS2012-3611 and No. CSD2007-00046 (Consolider NanoLight) and by the Comunidad de Madrid Microseres Project No. S2009/TIC1476.

\section{References}

[1] A. Ashkin, Acceleration and Trapping of Particles by Radiation Pressure, Phys. Rev. Lett. 24 (1970) 156159.
[2] A. Ashkin, J.M. Dziedzic, J. E. Bjorkholm, and S. Chu, Observation of a single-beam gradient force optical trap for dielectric particles, Opt. Lett. 11 (1986) 288-290.

[3] D.G. Grier, A revolution in optical manipulation, Nature 424 (2003) 810-816.

[4] K.C. Neuman and S.M. Block, Optical trapping, Rev. Sci. Instrum. 75 (2004) 2787-2895.

[5] M.M. Burns, J.-M. Fournier and J.A. Golovchenko, Optical Binding, Phys. Rev. Lett. 63 (1989) 12331236.

[6] R. Gómez-Medina, and J.J. Sáenz, Unusually Strong Optical Interactions between Particles in Quasi-OneDimensional Geometries, Phys. Rev. Lett. 93 (2004) 243602-243605.

[7] K. Dholakia and P. Zemánek, Gripped by light: optical binding, Rev. Mod. Phys. 82 (2010) 1767-1791.

[8] J. Chen, J. Ng, Z. Lin, and C. T. Chan, Optical pulling force, Nature Photonics 5 (2011) 531-534.

[9] J.J. Sáenz, Optical forces: Laser tractor beams, Nature Photon. 5 (2011) 514-515.

[10] A. Novitsky, C.-W Qiu and H. Wang, Single Gradientless Light Beam Drags Particles as Tractor Beams, Phys. Rev. Lett. 107 (2011) 203601-1-203601-4.

[11] S. Sukhov and A. Dogariu, Negative Nonconservative Forces: Optical Tractor Beams for Arbitrary Objects, Phys. Rev. Lett. 107 (2011) 203602-1-203602-4.

[12] A. Salandrino and D. N. Christodoulides, Reverse optical forces in negative index dielectric waveguide arrays, Opt. Lett. bf36 (2011) 3103-3105.

[13] A. Dogariu, S. Sukhov and J.J. Sáenz, Optically induced negative forces, Nat. Photon. 7 (2013) 24-27.

[14] O. Brzobohatý, V. Karásek, M. Šiler, L. Chvátal, T. Čižmár and P. Zemánek, Experimental demonstration of optical transport, sorting and self-arrangement using a tractor beam, Nature Photonics 7 (2013) 123127.

[15] L. Novotny and B. Hecht, Principles of Nano-Optics, Cambridge University Press, Cambridge 2006.

[16] M. Nieto-Vesperinas, P.C. Chaumet and A. Rahmani, Near-field photonic forces, Phil. Trans. R. Soc. Lond. A 362 (2004) 719-737.

[17] V. Wong and M. Ratner, Gradient and nongradient contributions to plasmon-enhanced optical forces on silver nanoparticles, Physical Review B 73, (2006) 075416-1-075416-6. 
[18] S. Albaladejo, M. I. Marqués, M. Laroche and J. J. Sáenz, Scattering Forces from the Curl of the Spin Angular Momentum of a Light Field, Phys. Rev. Lett. 102 (2009) 113602-1-113602-4.

[19] M. V. Berry Optical currents J. Opt. A: Pure Appl. Opt. 11 (2009) 094001-094013.

[20] D. B. Ruffner and D. G. Grier, Comment on Scattering Forces from the Curl of the Spin Angular Momentum of a Light Field, Phys. Rev. Lett. 111 (2013) 059301.

[21] M. I. Marqués and J. J. Sáenz, Reply to comment on Scattering Forces from the Curl of the Spin Angular Momentum of a Light Field, Phys. Rev. Lett. 111 (2013) 059302.

[22] I. Iglesias and J. J. Sáenz, Scattering forces in the focal volume of high numerical aperture microscope objectives, Opt. Commun. 284 (2011) 2430-2436.

[23] I. Iglesias and J. J. Sáenz, Light spin forces in optical traps: comment on "Trapping metallic Rayleigh particles with radial polarization”, Opt. Express 20 (2012) 2832-2834.

[24] Q. Zhan, Trapping metallic Rayleigh particles with radial polarization: reply to comment, Opt. Express 20, (2010) 6058-6059.

[25] A. Bekshaev, K. Y. Bliokh, M. Soskin, Internal flows and energy circulation in light beams, Journal of Optics 13 (2011) 053001-1-053001-32.

[26] A. Hemmerich and T. W. Hänsch, Radiation pressure vortices in two crossed standing waves, Phys. Rev. Lett. 68, (1992) 1492-1495.

[27] S. Albaladejo, M. I. Marqués, F. Scheffold, and J. J. Sáenz, Giant enhanced diffusion of gold nanoparticles in optical vortex fields, Nano Lett. 9 (2009) 3527 3531 .

[28] I. Zapata, S. Albaladejo, J.M.R. Parrondo, J.J. Sáenz and F. Sols,. Deterministic Ratchet from Stationary Light Fields. Phys. Rev. Lett., 103 (2009) 130601-1130601-4.

[29] S. Albaladejo, M. I. Marqués and J. J. Sáenz, Light control of silver nanoparticle's diffusion, Opt. Express 19 (2011) 11471-11478.

[30] M. I. Marqués and J. J. Sáenz, Scattering forces and electromagnetic momentum density in crossed circularly polarized standing waves, Opt. Lett 37 (2012) 2787-2789; Opt. Lett 37 (2012) 4470.

[31] P. C. Chaumet and A. Rahmani, Electromagnetic force and torque on magnetic and negative-index scatterers Opt. Express 17 (2009) 2224-2234.
[32] M. Nieto-Vesperinas, J. J. Sáenz, R. Gómez-Medina and L. Chantada, Optical forces on small magnetodielectric particles, Opt. Express 18 (2010) 1142811443.

[33] R. Gómez-Medina, M. Nieto-Vesperinas and J. J. Sáenz, Nonconservative electric and magnetic optical forces on submicron dielectric particles, Phys. Rev. A 83 (2011) 033825-033832.

[34] See f.i. J. D. Jackson, Classical Electrodynamics, 3er ed., page 241 .

[35] K.Y. Bliokh, A. Y. Bekshaev and F. Nori, Dual electromagnetism: Helicity, spin, momentum and angular momentum, New Journal of Physics 15 (2013) 033026-033054.

[36] R.P. Cameron and S. M. Barnett, Electric-Magnetic symmetry and Noether's theorem, New Journal of Physics 14 (2012) 123019-123046.

[37] See f.i. J. D. Jackson, Classical Electrodynamics, 3er ed., page 605 .

[38] See f.i. L. Mandel and E. Wolf, Optical coherence and quantum optics, page 488 . 